\title{
DUAL NEURAL NETWORK FOR ADAPTIVE SLIDING MODE CONTROL OF QUADROTOR HELICOPTER STABILIZATION
}

\author{
Hana Boudjedir ${ }^{1}$, Fouad Yacef ${ }^{1}$, Omar Bouhali $^{1}$ and Nassim Rizoug ${ }^{2}$ \\ ${ }^{1}$ Automatic laboratory LAJ, Department of Automatic, Jijel University, Algeria. \\ hana_boudjedir@yahoo.fr, Yaceffouad@yahoo.fr, bouhali_omar@yahoo.fr \\ ${ }^{2}$ Mecatronic Laboratory, ESTACA School, Laval, France \\ nrizoug@yahoo.fr
}

\begin{abstract}
An adaptive sliding mode control based on two neural networks is proposed in this paper for Quadrotor stabilization. This approach presents solutions to conventional control drawbacks as chattering phenomenon and dynamical model imprecision. For that reason two ANN for each quadrotor helicopter subsystem are implemented in the control loop, the first one is a Single Hidden Layer network used to approximate on line the equivalent control and the second feed-forward Network is used to estimate the ideal corrective term. The main purpose behind the use of ANN in the second part of SMC is to minimize the chattering phenomena and response time by finding optimal sliding gain and sliding surface slope. The learning algorithms of the two ANNs (equivalent and corrective controller) are obtained using the direct Lyapunov stability method. The simulation results are given to highlight the performances of the proposed control scheme.
\end{abstract}

\section{KEYWORDS:}

Adaptive control, Artificial Neural Network, Lyapunov, Quadrotor, sliding mode control

\section{INTRODUCTION}

In the last years, unmanned aerial vehicles (UAV) have gained a strong interest. The recent advances in low-power embedded processors, miniature sensors and control theory are opening new horizons in terms of miniaturization and field of application [1]. The Quadrotor Helicopter is considered as one of the most popular UAV platform. The mains reasons for all this attention has stemmed from its simple construction and its large payload as compared with the conventional helicopter [1].

The dynamics of the Quadrotor are nonlinear and like the most flying robots, the Quadrotor helicopter is characterized by its under actuated nature which can make the difficult to control. The Quadrotor has six degrees of freedom and only four control inputs. To solve the Quadrotor UAV tracking control problem many techniques have been proposed [2, 3, 4, 5, 6, and 7] where the control objective is to tack three desired cartesian positions and a desired yaw angle. 
Many approaches in the literature that use different control techniques have dealt with control of the Quadrotor. Nonlinear Backstepping and sliding mode controls have been proposed in [2], [3]. In [4] the Hळ type robust control law was used, in [5] a PID control has been applied.

The drawback of all these techniques is the need for nonlinear dynamics exactly knows. However the adaptive control presents a solution to the problems met by the classic schemas. Nevertheless this technique is only applicable in two cases: if the model is linear as in [8] or if the unknown parameters are assumed to appear linearly with respect to know nonlinear functions [9]. However this assumption is not sufficient for many piratical situations; because it is difficult to describe a nonlinear plant by know nonlinear functions precisely [9].

Known by their big capacities in approximation, neural networks have been used with success in many adaptive control schemas. Several kinds of Neural Networks adaptive schemes have been proposed in the literature for Quadrotor's control [6, 7, 10, 11, 12, and 13].

Sliding mode control (SMC) is an important robust control approach. This technique provides a systematic approach for a large class of systems to maintaining stability problem and consistent performance in the face of modelling imprecision.

However this control technique has three drawbacks where two of them are caused by the corrective term and the third is imposed by the equivalent term. As is known the main disadvantage of SMC is the chattering phenomena, the second one is the choice of the sliding gain; the implementation of the switching signal requires the determination of the ideal sliding gain that depends on parametric and external disturbances, which is difficult if not impossible. The third disadvantage of the SMC is the need to have even partial knowledge of the system dynamics. In this context, several methods based on fuzzy logic [14, 15], and Neural Network [16] has been proposed.

The present paper propose a new robust adaptive control that does not require any prior information on model dynamics based on the use of tow Neural Networks in parallel in the control loop to Quadrotor stabilization. The first ANN is a SHL used to compute on line the nonlinear equivalent control. To overcome the corrective term drawbacks a second feed-forward $\mathrm{NN}$ is added to the adaptive control scheme in which its principal objective is to reduce chattering phenomena and to approximate the optimal sliding gain and sliding surface slope in order to minimize the response time. The design methodology is based on the Lyapunov stability. A Quadrotor Helicopter is utilized to perform the control effect of the designed Neural sliding mode controller.

This paper is organized as follows. In Section 2, the dynamical model of the Quadrotor will be described. In section 3, Problem formulation is presented. In Section 4, adaptive neural network control based on sliding mode technique for Quadrotor helicopter control will be developed. Section 5, simulation results are given to show the effectiveness and feasibility of the proposed scheme. The conclusion is given in section 6 .

\section{QUADRotor Modelling}

The Quadrotor is an air vehicle with four thrusters mounted at the ends of a cross. See. Figure.1 


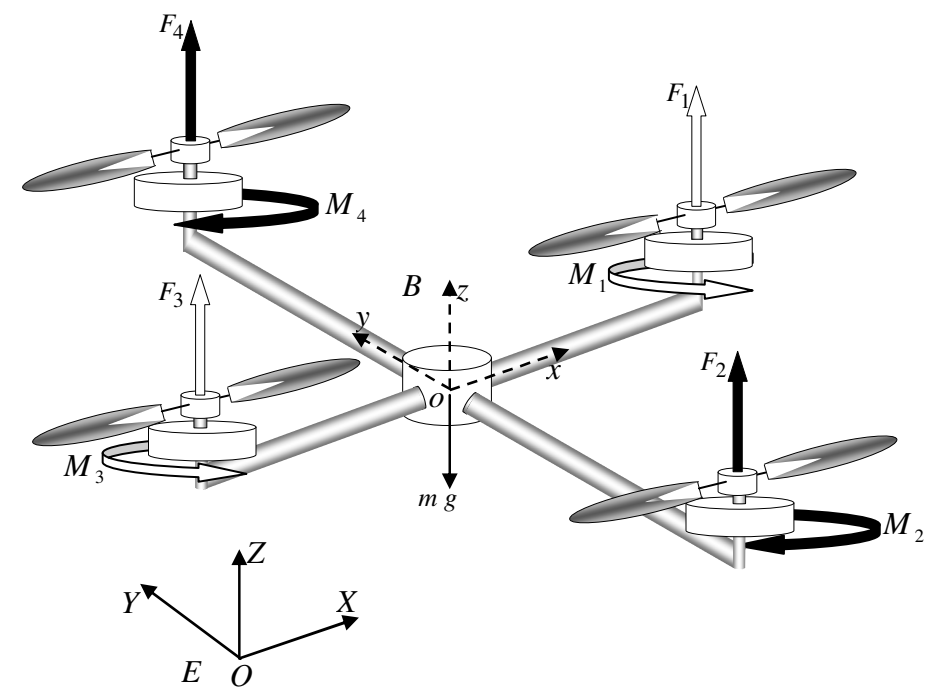

Figure 1. Model of conventional Quadrotor.

The front and rear motors $(1,3)$ in conventional Quadrotor rotate in the counterclockwiseclockwise, and the lateral motors $(2,4)$ rotate in the opposite direction, in order to avoid turning the Quadrotor on itself.

Each rotor, provide a vertical force responsible on the lift and the motions of the helicopter. Usually it exists three mode of flight according to rotation velocity of the motors. Vertical flight ascending (descending) is obtained by the increase (decrease) of the Thrust forces to getting a difference from the weight of the Quadrotor. Pitching motion in a Quadrotor is the result of the speed difference between front and rear rotors, hence a rotation around the $Y$ axis coupled with a translation along the $X$ axis are produced. The same analogy is applied to obtain the roll motion, but this time by changing the side motors speed. The yaw motion is obtained while increasing (decreasing) speed of motors (1.3) compared with that motors (2.4). Unlike pitch and roll motions, yaw rotation is the result of reactive torques produced by the rotation of the rotors.

By using the formalism of Newton-Euler [2, 3, 4, 5, 6, and 9] the dynamic model of a Quadrotor can be expressed as:

$$
\left\{\begin{array}{l}
\ddot{\varphi}=\frac{U_{\varphi}(t)}{I_{x}}+\frac{\left(I_{y}-I_{z}\right)}{I_{x}} \dot{\psi} \dot{\theta}-\frac{J_{r} \Omega_{r}(t)}{I_{x}} \dot{\theta}-\frac{k_{f r x}}{I_{x}} \dot{\varphi}^{2}+d_{\varphi} \\
\ddot{\theta}=\frac{U_{\theta}(t)}{I_{y}}+\frac{\left(I_{z}-I_{x}\right)}{I_{y}} \dot{\psi} \dot{\varphi}+\frac{J_{r} \Omega_{r}(t)}{I_{y}} \dot{\varphi}-\frac{k_{f r y}}{I_{y}} \dot{\theta}^{2}+d_{\theta} \\
\ddot{\psi}=\frac{U_{\psi}(t)}{I_{z}}+\frac{\left(I_{x}-I_{y}\right)}{I_{z}} \dot{\varphi} \dot{\theta}-\frac{k_{f r z}}{I_{z}} \dot{\psi}^{2}+d_{\psi} \\
\ddot{x}=\frac{1}{m}\left(\left(C_{\psi} S_{\theta} C_{\varphi}+S_{\varphi} S_{\psi}\right) U_{1}(t)-k_{f f x} \dot{x}\right)+d_{x} \\
\ddot{y}=\frac{1}{m}\left(\left(S_{\psi} S_{\theta} C_{\varphi}-S_{\varphi} C_{\psi}\right) U_{1}(t)-k_{f f y} \dot{y}\right)+d_{y} \\
\ddot{z}=\frac{1}{m}\left(\left(C_{\theta} C_{\varphi}\right) U_{1}(t)-k_{f t z} \dot{z}\right)-g+d_{z}
\end{array}\right.
$$


Where : $m$ : Quadrotor mass, $k_{p}$ : Thrust factor, $k_{d}:$ drag factor, $\omega_{i}:$ angular rotor speed, $J=\operatorname{diag}\left(I_{x}\right.$, $\left.I_{y}, I_{z}\right)$ : inertia matrix, $K_{f t}=\operatorname{diag}\left(k_{f t x}, k_{f t y}, k_{f z z}\right)$, drag translation matrix, $K_{f r}=\operatorname{diag}\left(k_{f r x}, k_{f r y}, k_{f r z}\right)$ : friction aerodynamic coefficients, $\xi=\left[\begin{array}{lll}x & y & z\end{array}\right]^{T}$ : position vector, $\eta=\left[\begin{array}{lll}\varphi & \theta & \psi\end{array}\right]^{T}$ represents the angles of roll, pitch and yaw, $d_{x, y, z, \varphi, \theta, \psi}$, disturbance and $\Omega_{r}=\sum_{i=1}^{4}(-1)^{i+1} \omega_{i}$.

The control inputs according to the angular velocities of the four rotors are given by:

$$
\left[\begin{array}{l}
U_{1}(t) \\
U_{\varphi}(t) \\
U_{\theta}(t) \\
U_{\psi}(t)
\end{array}\right]=\left[\begin{array}{cccc}
k_{p} & k_{p} & k_{p} & k_{p} \\
0 & -l k_{p} & 0 & l k_{p} \\
-l k_{p} & 0 & l k_{p} & 0 \\
k_{d} & -k_{d} & k_{d} & -k_{d}
\end{array}\right]\left[\begin{array}{c}
\omega_{1}^{2} \\
\omega_{2}^{2} \\
\omega_{3}^{2} \\
\omega_{4}^{2}
\end{array}\right]
$$

\subsection{Virtual control}

To ensure that every Quadrotor output follows a specified trajectory, we will introduce those virtual control input [13]:

$$
\left\{\begin{array}{l}
U_{x}(t)=\left(C_{\psi} S_{\theta} C_{\varphi}+S_{\varphi} S_{\psi}\right) U_{1}(t) \\
U_{y}(t)=\left(S_{\psi} S_{\theta} C_{\varphi}-S_{\varphi} C_{\psi}\right) U_{1}(t) \\
U_{z}(t)=\left(C_{\theta} C_{\varphi}\right) U_{1}(t)
\end{array}\right.
$$

The physical interpretation of theses virtual control, means that the control of translation motion depends on three common inputs are: $\theta, \varphi$ and $U_{l}(t)$. This requires that the rolling and pitching motions must take a desired trajectory to guarantee the control task of translation motion. Using equation (3) the desired trajectories in rolling and pitching are defined as follow [13]:

$$
\left\{\begin{array}{l}
\varphi_{d}=\arcsin \left(\frac{U_{x}(t) S_{\psi d}-U_{y}(t) C_{\psi d}}{\sqrt{U_{x}^{2}(t)+U_{y}^{2}(t)+U_{z}^{2}(t)}}\right) \\
\theta_{d}=\arctan \left(\frac{C_{\psi d} U_{x}(t)+S_{\psi d} U_{y}(t)}{U_{z}(t)}\right)
\end{array}\right.
$$

with $\varphi_{d}, \theta_{d}$ and $\psi_{d}$ are the desired trajectories in roll, pitch and yaw respectively.

\section{Problem Formulation}

After the implantation of the virtual control defined in equation (3), the quadrotor helicopter dynamic model is the nonlinear time-varying system described by two differentials equations with the following structure for each one:

$$
\ddot{Y}=F(X)+G(X) U
$$

where: $X=\left[\begin{array}{llll}y_{1} & \dot{y}_{1} & y_{2} & \dot{y}_{2}, \cdots, \dot{y}_{p}\end{array}\right]^{T}$ is the output which is assumed available for measurement, $U=\left[\begin{array}{lll}u_{1} & \cdots & u_{p}\end{array}\right]^{T}$ is the input vector, $Y=\left[\begin{array}{lll}y_{1} & \cdots & y_{p}\end{array}\right]^{T}$ is the output vector and $F(X), G(X)$ are smooth nonlinear functions. 
Assumption.1: The desired output trajectory $Y_{d}(t)$ and its first derivative are smooth and bounded.

Assumption.2: The gain $\mathrm{G}(X)$ is bounded, positive definite and slowly time varying.

Let's define the tracking error as:

$$
e(t)=Y_{d}(t)-Y(t)
$$

and the filtered tracking errors by [9]:

$$
S=\dot{e}+\lambda(t) e
$$

Where : $S=\left[\begin{array}{lll}s_{1} & \cdots & s_{p}\end{array}\right]^{T}$ and $\lambda(t) \in \mathfrak{R}^{p \times p}>0$ is a diagonal matrix.

From (16), we deduce that the convergence of $S$ to zero implies convergence of the tracking error and its derivative to zero [9]. So, the objective of control is summarized in the synthesis of a control law that allows the convergence to zero of the filtered error.

The filtered error first time derivative is giving by:

$$
\dot{S}=v-F(X)-G(X) U
$$

Where:

$$
v=\ddot{Y}_{d}+\lambda(t) \dot{e}+\dot{\lambda}(t) e
$$

The ideal sliding mode control (SMC) who guaranteed the closed loop system performances may be given by:

$$
U^{*}=U_{e q}^{*}+U_{c o r}^{*}
$$

$\stackrel{\leftrightarrow}{\leftrightarrow} U_{e q}^{*}:$ is the ideal equivalent control,

$$
U_{e q}^{*}=G^{-1}(v-F)
$$

$\stackrel{H_{c o r}}{*}:$ is the ideal corrective control:

$$
U_{\text {cor }}^{*}=K^{*} \operatorname{sign}\left(S^{*}\right)
$$

Where : $K^{*}$, present the sliding gain optimal value and $S^{*}$ is the sliding surface with ideal slope $\lambda^{*}(t)$.

Substituting (10), (11) and (12) in (8), filtered error dynamics becomes:

$$
\dot{S}=-G K^{*} \operatorname{sign}\left(S^{*}\right)
$$

implying that $S \rightarrow 0$ and therefore $e, \dot{e} \rightarrow 0 \quad j=\overline{1: r_{i}-1}, i=\overline{1: p}$ [9]. 
However, the implementation of the control law defined in equation (10) is impossible for the following reasons:

(i) The dynamical mode is completely unknown,

(ii) The discontinuous term will introduce high frequency signals that may excite the unmodelled dynamics and deterioration of the controlled system,

(iii) The optimal value of the gain is unknown because it depends on parametric and external disturbances.

So our goal is to use artificial neural networks in order to solve problems encountered by the classical sliding mode technique

\section{DESIGN OF ADAPTIVE CONTROL}

\subsection{Equivalent control}

In this section, the approximation of the corresponding command defined in (10) will be performed by SHL Networks (Figure.2). According to universal approximation theorem, the neural network of the type SHL can estimate any nonlinear, continuous, unknown function [12]. So that that ideal estimation of the equivalent controller presented in (11) by a SHL is giving by the following expression:

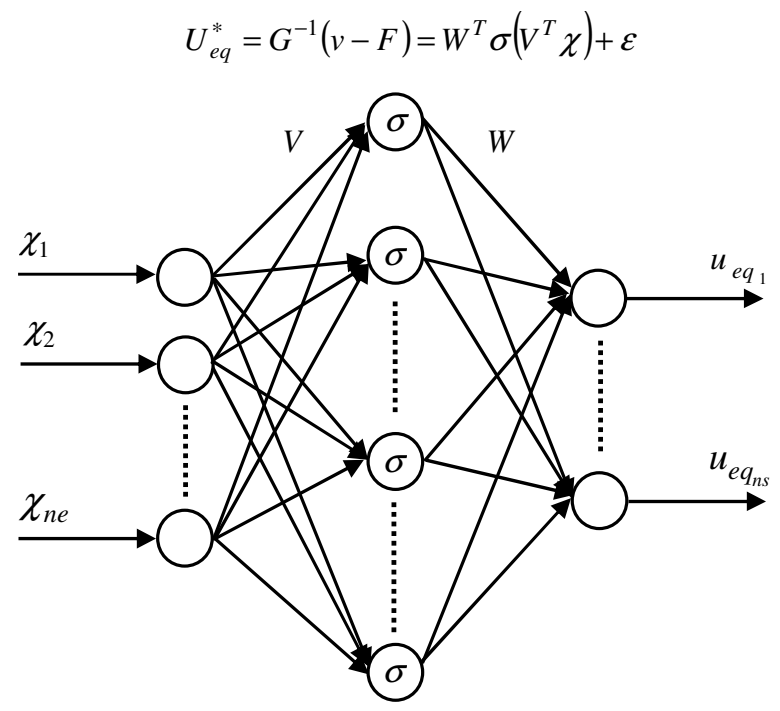

Figure 2: SHL NN used in the control schemes.

where: ne input neurons number, $n c$ hidden neurons number, $n s=3$ output neurons number, $\chi \in \mathfrak{R}^{n e}$ input vector and $\sigma()$ is sigmoid activation function, $W \in \mathfrak{R}^{n c \times n s}$ and $V \in \mathfrak{R}^{n e \times n c}$ are the ideal weight and $\varepsilon$ is the reconstruction error.

The optimal weights are unknown, it is necessary to estimate them by an adaptation mechanism so that the output feedback control law can be realized.

The matrixes $\hat{W}$ and $\hat{V}$ are the estimate of $W$ and $V$ respectively. Thus, the adaptive control approximating the ideal SHLNN output defined in (14) is given by : 
International Journal of Information Sciences and Techniques (IJIST) Vol.2, No.4, July 2012

$$
U_{e q}=\hat{W}^{T} \sigma\left(\hat{V}^{T} \chi\right)
$$

Assumption.3: $\|W\|_{F} \leq W_{m},\|V\|_{F} \leq V_{m}$. with : $W_{m}$ et $V_{m}$ are unknown positive constants.

In this case, the equivalent control identification error is giving by :

$$
\tilde{U}_{e q}=U_{e q}^{*}-U_{e q}=\tilde{W}^{T} \hat{\sigma}+\hat{W}^{T} \hat{\sigma}^{\prime} \tilde{V}^{T} \chi+w
$$

Where : $\tilde{W}=W-\hat{W}, \tilde{V}=V-\hat{V}$ are parameter estimation errors and $w$ presents the estimation errors:

$$
w=\varepsilon+\tilde{W}^{T} \hat{\sigma}^{\prime} \tilde{V}^{T} \chi+W^{T} O\left(\tilde{V}^{T} \chi\right)^{2}
$$

Assumption.4: $\|w\| \leq \bar{w}$. with : $\bar{w}$ is an unknown positive constant.

To achieve the goal of controlling the weights adaptation laws based on $e$-modification technique are defined by:

$$
\left\{\begin{array}{l}
\dot{\hat{W}}=F_{W}\left(\hat{\sigma} S^{T}-\kappa\|S\| \hat{W}\right) \\
\dot{\hat{V}}=F_{V}\left(\chi S^{T} \hat{W}^{T} \hat{\sigma}^{\prime}-\kappa\|S\| \hat{V}\right)
\end{array}\right.
$$

with: $F_{W}>0, F_{V}>0$ are the adaptive gains and $\kappa$ is a positive constant.

\subsection{Corrective control}

As we already note, the corrective control in SMC is characterized by the charting phenomena provide by the discontinuous term, for that reason we will replace it by a continuous function $T($.) giving by:

$$
T(z)=\frac{1-e^{-2 z}}{1+e^{-2 z}}
$$

So the ideal expression of corrective term will be:

$$
U_{\text {cor }}^{*}=K^{*} T\left(S^{*}\right)
$$

A second neural network is added to the control scheme. The purpose here is estimating the ideal corrective term including the ideal sliding gain and surface. The net used is shown on Figure. 3. 


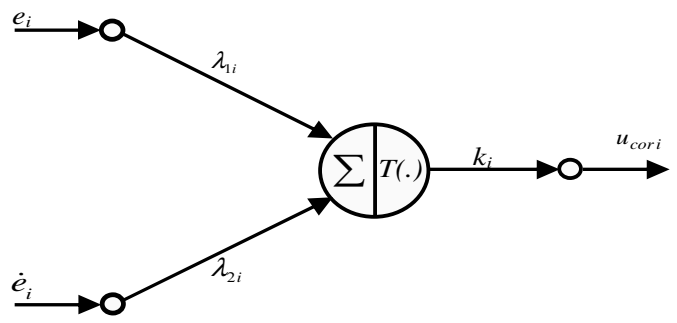

Figure. 3. The network used to estimate corrective control.

The ideal output of such network is defined by:

$$
u_{c o r i}^{*}=k_{i} T\left(\Gamma_{i}^{T} \chi_{i}^{e}\right)+\varepsilon_{g_{i}} \quad i=\overline{1: p}
$$

Where : the gain $k_{i}$ and $\Gamma_{i}=\left[\begin{array}{ll}\lambda_{1 i} & \lambda_{2 i}\end{array}\right]^{T}$ present the ideal output layer and hidden layer weight respectively, $\chi_{i}^{e}=\left[\begin{array}{ll}e_{i} & \dot{e}_{i}\end{array}\right]^{T}$ is the input vector, $\varepsilon_{g_{i}}$ is the reconstruction error and $T($.$) is the$ activation function defined in equation (20).

The weights $k_{i}$ and $\Gamma_{i}=\left[\begin{array}{ll}\lambda_{1 i} & \lambda_{2 i}\end{array}\right]^{T}$ are unknown, so it is judicious to find a way for adapting them. So the real estimate of the corrective term by the ANN presented in Figure. 3 has the following structure:

$$
u_{\text {cori }}=\hat{k}_{i} T\left(\hat{\Gamma}_{i}^{T} \chi_{i}^{e}\right) \quad i=\overline{1: p}
$$

The corrective term estimation error is given by:

$$
\tilde{u}_{\text {cor } i}=\tilde{k}_{i} \hat{T}_{i}+\hat{k}_{i} \hat{T}_{i}^{\prime} \tilde{\Gamma}_{i}^{T} \chi_{i}^{e}+w_{g_{i}} \quad i=\overline{1: p}
$$

Where: $\hat{k}_{i}, \hat{\Gamma}_{i}$ are the estimate of $k_{i}, \Gamma_{i}$ respectively, and $\tilde{k}_{i}=k_{i}-\hat{k}_{i}, \tilde{\Gamma}_{i}=\Gamma_{i}-\hat{\Gamma}_{i}$ are the parameter errors. The term $w_{g_{i}}$ present the approximation error given by:

$$
w_{g_{i}}=\varepsilon_{g_{i}}+\tilde{k}_{i} \hat{T}_{i}^{\prime} \hat{\Gamma}_{i}^{T} \chi_{i}^{e}+\tilde{k}_{i} O\left(\tilde{\Gamma}_{i}^{T} \chi_{i}^{e}\right)^{2}
$$

Assumption 5: $\left\|w_{g_{i}}\right\| \leq \bar{w}_{g_{i}}, \bar{w}_{g_{i}}$ is an unknown positive constant and $\left|k_{i}\right| \leq k_{m_{i}},\left\|\Gamma_{i}\right\|_{F} \leq \Gamma_{m_{i}}$. with : $\Gamma_{m i}$ and $k_{m i}$ are unknown positive constants.

The proposed adaptation laws for adjusting weights are giving by the following equations:

$$
\left\{\begin{array}{l}
\dot{\hat{k}}_{i}=F_{k_{i}} \hat{T}\left(\Gamma_{i}^{T} \chi_{i}^{e}\right) s_{i} \\
\dot{\hat{\Gamma}}_{i}=F_{\Gamma_{i}}\left(\chi_{i}^{e} s_{i} \hat{k}_{i} \hat{T}^{\prime}+\kappa_{\Gamma i}\left|s_{i}\right|\left(\bar{\Gamma}_{i}-\hat{\Gamma}_{i}\right)\right)
\end{array}\right.
$$

Where : $F_{k_{i}}=F_{k_{i}}^{T}>0, F_{\Gamma_{i}}=F_{\Gamma_{i}}^{T}>0, \kappa_{\Gamma_{i}}>0$. 
The vector $\bar{\Gamma}_{i}$ is selected as follows:

$$
\bar{\Gamma}_{i}=\left[\frac{c_{i}}{\left|e_{i}\right|+\varepsilon_{i}} 1\right]^{T}, c_{i}, \varepsilon_{i}>0
$$

This expression implies can rotate in the phase space the surface rotation according to the tracking error in order to reduce the response time and filtered error, where:

$\Leftrightarrow$ The slope is small, if the tracking error is large as in Figure.4.a,

$\Leftrightarrow$ The slope is large, if the tracking error is small as in Figure.4.b.

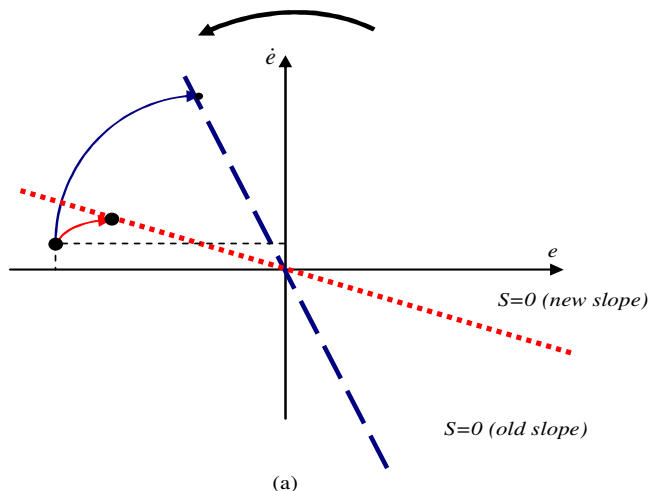

(a)

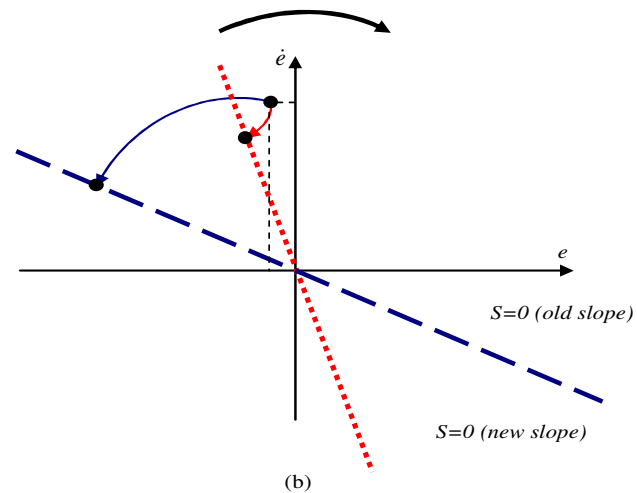

(b)

Figure 4: sliding surface rotation.

\subsection{Proof}

Consider the Lyapunov function defined by :

$$
L=\frac{1}{2} S^{T} G^{-1} S+\frac{1}{2} \operatorname{tr}\left(\tilde{W}^{T} F_{W}^{-1} \tilde{W}\right)+\frac{1}{2} \operatorname{tr}\left(\tilde{V}^{T} F_{V}^{-1} \tilde{V}\right)+\frac{1}{2} \sum_{i=1}^{p}\left\{F_{k_{i}}^{-1}\left(\tilde{k}_{i}\right)^{2}+\left(\tilde{\Gamma}_{i}\right)^{T} F_{\Gamma_{i}}^{-1}\left(\tilde{\Gamma}_{i}\right)\right\}
$$

The first time derivative of (27) is :

$$
\dot{L}=S^{T} G^{-1} \dot{S}+\operatorname{tr}\left(\tilde{W}^{T} F_{W}^{-1} \dot{\tilde{W}}\right)+\operatorname{tr}\left(\tilde{V}^{T} F_{V}^{-1} \dot{\tilde{V}}\right)+\sum_{i=1}^{p}\left\{F_{k_{i}}^{-1}\left(\tilde{k}_{i}\right)\left(\dot{\tilde{k}}_{i}\right)+\left(\tilde{\Gamma}_{i}\right)^{T} F_{\Gamma_{i}}^{-1}\left(\dot{\tilde{\Gamma}}_{i}\right)\right\}
$$

The filtered error dynamic in equation (8), can be written as :

$$
\dot{S}=-G\left(U_{c o r}^{*}-\tilde{U}_{a d}-\tilde{U}_{c o r}\right)
$$

Where

$$
U_{\text {cor }}^{*}=\left[\begin{array}{lll}
u_{\text {cor } 1}^{*} & \cdots & u_{c o r p}^{*}
\end{array}\right]^{T}
$$

The implementation of (18), (25), (29) in (28), we will have: 


$$
\dot{L} \leq-S^{T} U_{c o r}^{*}+\|S\|\left(\bar{w}+\kappa \frac{W_{m}^{2}+V_{m}^{2}}{4}\right)+\sum_{i=1}^{p}\left\{\left(\bar{w}_{g_{i}}+\kappa_{\Gamma i} \frac{\Gamma_{m i}^{2}}{4}-\kappa_{\Gamma i}\left\|\tilde{\Gamma}_{i}\right\| \mid \bar{\Gamma}_{i} \|\right)\left|s_{i}\right|\right\}
$$

Suppose that: $S^{T} U_{c o r}^{*}$ is sufficiently large to compensate the other positives term in equation (31). So the Lyapunov first time derivative will be:

$$
\dot{L} \leq 0
$$

Which guarantee stability of closed-loop system [9].

\section{SIMULATION RESULTS}

In the simulation two SHL (translation And rotation motion) are implemented in order to estimate the equivalent controller, each network consists of three neurons in output layer, three hidden neurons and five neurons in the input layer where the vector: $\chi_{i}=\left[\begin{array}{lllll}\ddot{y}_{d i} & \dot{y}_{d i} & y_{d i} & e_{i} & \dot{e}_{i}\end{array}\right]^{T}$.

Figure. 5 shows the performance of this approach in the presence of a sinusoidal external perturbation [11] giving in equation (33) and a parametric variation of order 50\% from time $t=$ $10 \mathrm{sec}$

$$
\left\{\begin{array}{l}
d_{x}=d_{y}=d_{z}=0.3 \sin (t)+0.3 \\
d_{\varphi}=d_{\theta}=d_{\psi}=0.5 \sin (t)+0.5
\end{array}\right.
$$

The simulation parameters used are:

Table 1. Quadrotor Parameters

\begin{tabular}{cccc}
\hline Symbol & Value & Symbol & Value \\
\hline $\mathrm{m}$ & $0.486(\mathrm{Kg})$ & $\mathrm{I}_{\mathrm{z}}$ & $7.65 \mathrm{e}-3\left(\mathrm{KgM}^{2}\right)$ \\
1 & $0.25(\mathrm{~m})$ & $\mathrm{g}_{\mathrm{ft}}$ & $9.81\left(\mathrm{Ms}^{2}\right)$ \\
$\mathrm{k}_{\mathrm{d}}$ & $3.23 \mathrm{e}-7\left(\mathrm{NMs}^{2}\right)$ & $\mathrm{k}_{\mathrm{ffx}}, \mathrm{k}_{\mathrm{fty}}$ & $5.56 \mathrm{e}-4(\mathrm{Ns} / \mathrm{M})$ \\
$\mathrm{k}_{\mathrm{p}}$ & $2.98 \mathrm{e}-5\left(\mathrm{Ns}^{2}\right)$ & $\mathrm{k}_{\mathrm{ftz}}$ & $6.35 \mathrm{e}-4(\mathrm{Ns} / \mathrm{M})$ \\
$\mathrm{Jr}$ & $2.83 \mathrm{e}-5\left(\mathrm{KgM}^{2}\right)$ & $\mathrm{k}_{\mathrm{fr}}, \mathrm{k}_{\mathrm{fr} \theta}$ & $5.56 \mathrm{e}-4(\mathrm{Ns} / \mathrm{r})$ \\
$\mathrm{I}_{\mathrm{x}}$ & $3.82 \mathrm{e}-3\left(\mathrm{KgM}^{2}\right)$ & $\mathrm{k}_{\mathrm{fr}}$ & $6.35 \mathrm{e}-4(\mathrm{Ns} / \mathrm{r})$ \\
$\mathrm{I}_{\mathrm{y}}$ & $3.82 \mathrm{e}-3\left(\mathrm{KgM}^{2}\right)$ & & \\
\hline
\end{tabular}

Table 2: Controller Parameters.

\begin{tabular}{cccc}
\hline Symbol & Value & Symbol & Value \\
\hline$F_{W \xi, \eta}$ & $10 I_{3.3}$ & $c_{z}$ & 0.5 \\
$F_{V \xi, \eta}$ & $10 I_{15.15}$ & $c_{\varphi, \theta}$ & 0.2 \\
$\kappa_{\xi, \eta}$ & 1 & $c_{\psi}$ & 1 \\
$F_{k x, y}$ & 100 & $F_{\Gamma x, y}$ & $\operatorname{Diag}(2,0)$ \\
$F_{k z}$ & 200 & $F_{\Gamma z}$ & $\operatorname{Diag}(5,0)$ \\
$F_{k \varphi, \theta, \psi}$ & 50 & $F_{\Gamma \varphi, \theta}$ & $\operatorname{Diag}(20,0)$ \\
$\varepsilon_{\varphi, \theta, \psi}$ & 0.01 & $F_{\Gamma \psi}$ & $\operatorname{Diag}(10,0)$ \\
$\varepsilon_{x, y}$ & 0.3 & $\kappa_{\Gamma, y}$ & 10 \\
$\varepsilon_{z}$ & 0.1 & $\kappa_{\Gamma z}$ & 1 \\
$c_{x, y}$ & 1 & $\kappa_{\Gamma \varphi, \theta, \psi}$ & 0.5 \\
\hline
\end{tabular}


International Journal of Information Sciences and Techniques (IJIST) Vol.2, No.4, July 2012

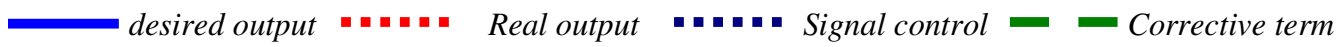
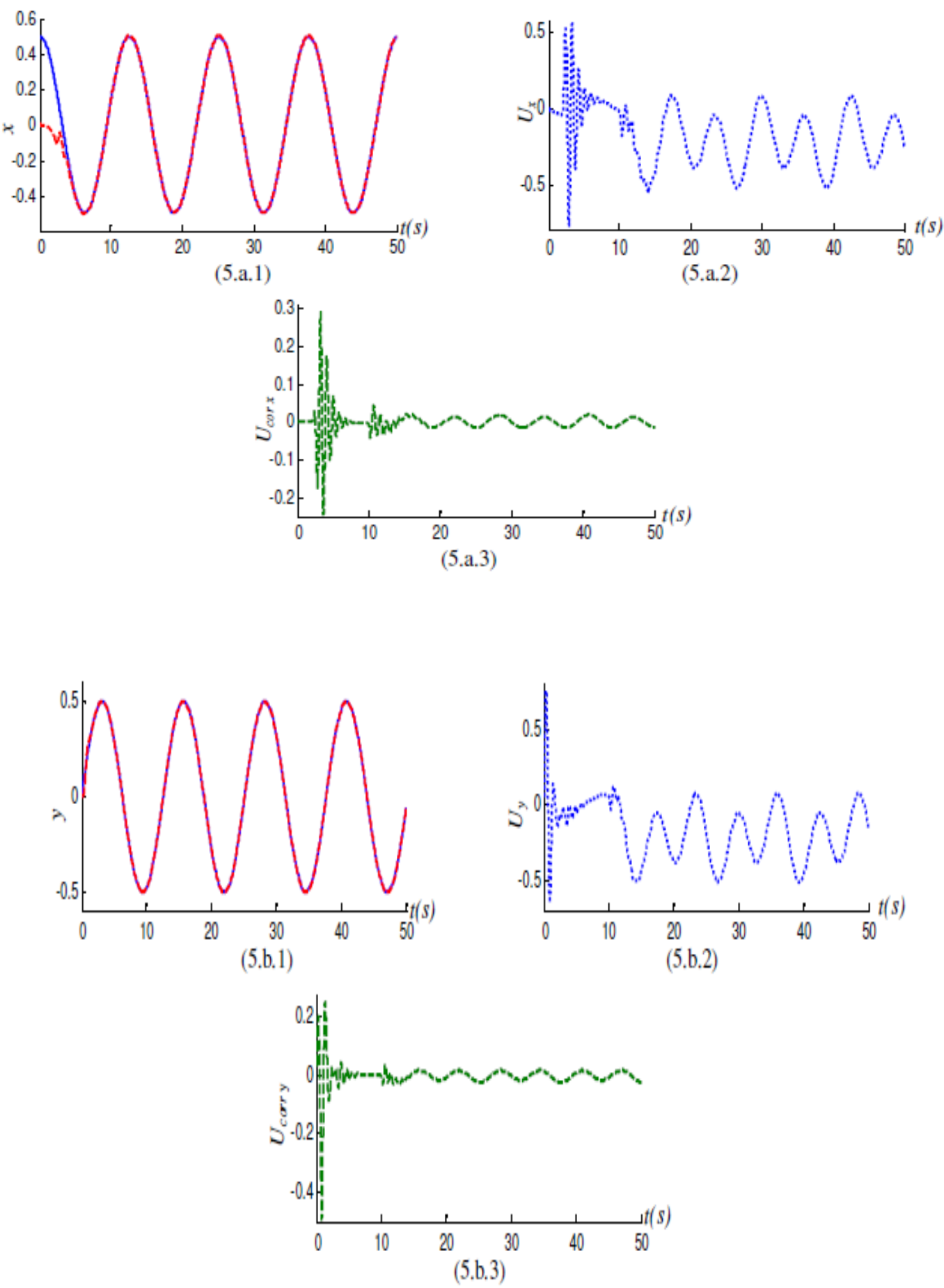

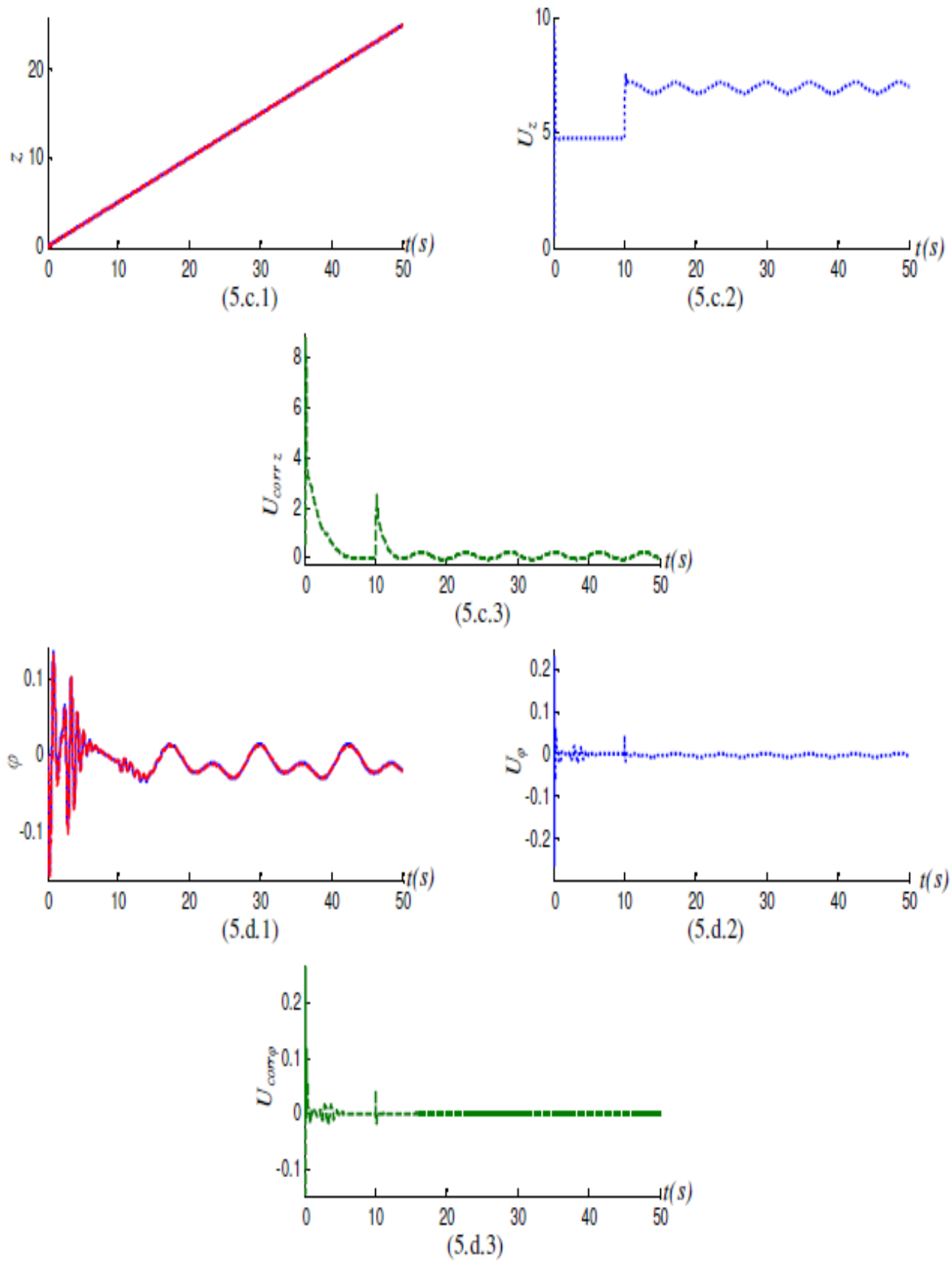

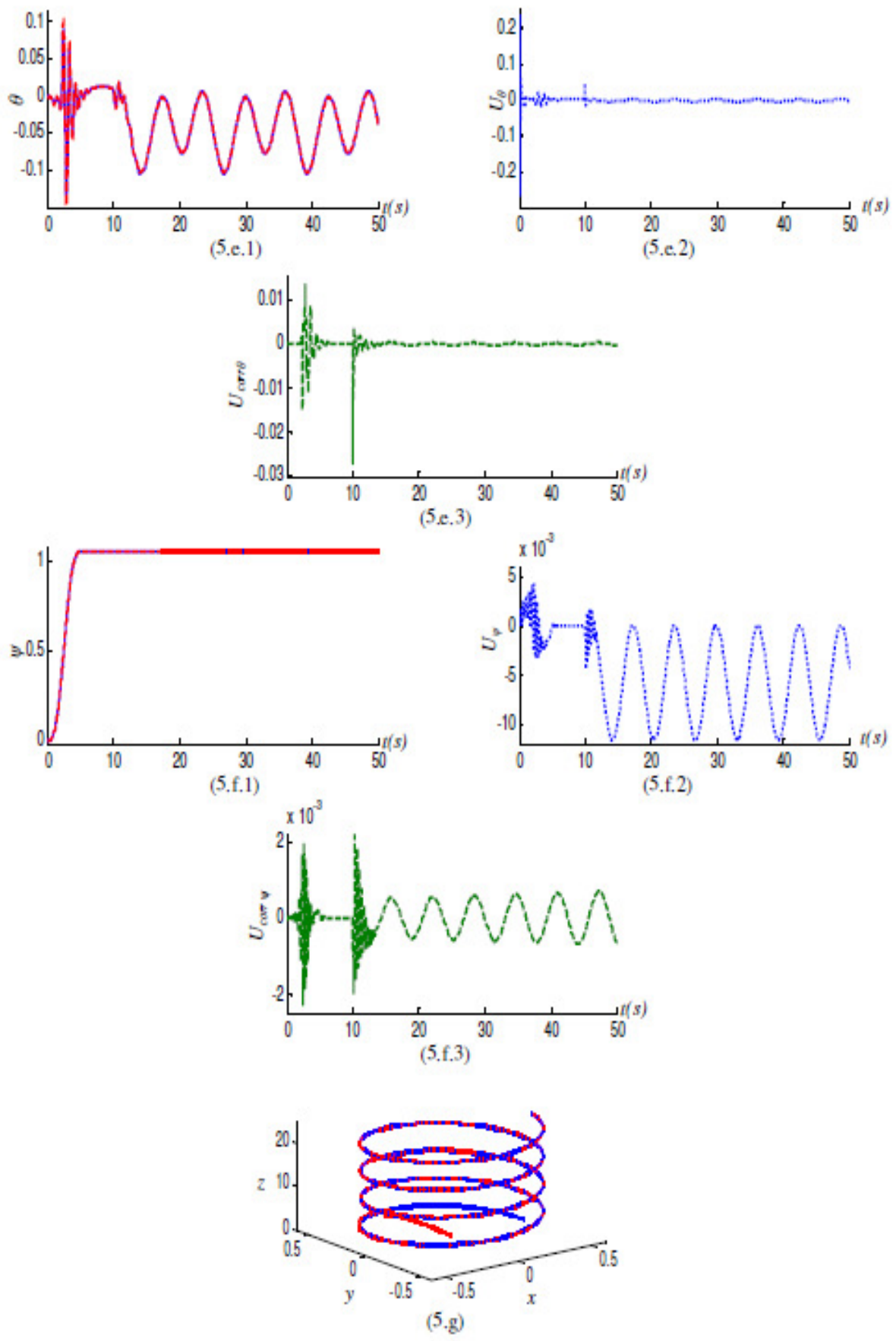

Figure.5: simulation result 
According to simulation result, the proposed approach appears sufficient, where the actual trajectory converges to the desired trajectory, even when the parametric and external disturbances are present.

The use of the ANN presented on Figure. 3 to estimate the corrective term has permitted to eliminated the chattering phenomena as well as allow us to avoid the adjustment of sliding gain without any performance degradation.

\section{Conclusion}

A neural network adaptive control based on sliding mode technique for a class of unknown nonlinear MIMO system is proposed in this paper. The scheme is realized by two parallel ANN where one of them is a SHL NN responsible on the equivalent controller accounting. The second one is a feed-forward NN used in order to solve the corrective term problems as chattering phenomena and the ideal value of sliding gain. The proposed method does not require any prior knowledge about dynamic model or/ and parametric and external disturbances. A stability study is performed by the direct method of Lyapunov. The performances of the proposed control scheme were validated by simulation of a control Quadrotor.

\section{REFERENCES}

[1] S. Bouabdallah \& al: Autonomous Miniature Flying Robots: Coming Soon. Robotics and Automation Magazine, pp 1-8, 2006.

[2] T. Madani \& A.Benallegue: Backstepping Control with Exact 2-Sliding Mode Estimation for a Quadrotor Unmanned Aerial Vehicle. Proc of IEEE/RSJ International CIRS, 2007.

[3] S.Bouabdallah \& R. Siegwart : Backstepping and sliding mode Techniques Applied to an Indoor Micro Quadrotor. In Proc 2005 IEEE International Conference on robotics and automation, 2005.

[4] A.Mokhtari, A.Benallegue and B.Daachi: Robust Feedback Linearization and GHळ Controller for a Quadrotor Unmanned aerial vehicle. Journal of Electrical Engineering, vol.57, No.1, pp 20.27, 2006.

[5] S.Bouaddallah, A.Noth \& R.Siegwart: PID vs. LQ control Techniques Applied to an Indoor Micro Quadrotor. In Proc IEEE international conference on intelligent Robots and systems, Japan, 2004.

[6] T.Dieks and S.Jagannathan : Neural Networks Out Feedback of a Quadrotor UAV. In Proc IEEE, 47th Conference on decision and control, pp 3633-3639, Cancun Mexico, Dec 2008.

[7] A.Das, F.Lewis and K.Subbarao : Backstepping Approach for Controlling a Quadrotor Using Lagrange Form Dynamics. J Intell Robot syst, pp 127-151, 2009.

[8] P.A.Ioannou : Robust adaptive control, ACC, pp 1574-1578, 1984.

[9] J.J.Slotine : Applied nonlinear control. Prentice Hall, 1991.

[10] K.Zemalache \& H.Maaref : Contolling a drone : comparison between a based model method and a fuzzy inference system. Applied soft Computing, pp 553-562, 2009.

[11] C. Nicol, CJ.B. Macnab \& A.R.Serrano: Robust Neural Network of a Quadrotor Helicopter, in proc IEEE Fuzzy information PSC, pp. 454-458, 2008.

[12] J.C.Raimundez \& A.F.Villaverde : Adaptive Tracking control for a Quad-rotor. ENOC-2008, saint Pertersburg, Russia July 2008.

[13] O.Bouhali and H.Boudjedir : Neural Network control with neuro-sliding mode observer applied to Quadrotor Helicopter. In Proc INISTA’11, pp 24-28, Istanbul, Turkey 2011.

[13] Ching-Chang Wong and al : Genetic-based Sliding Mode Fuzzy Controller Design. Journal of Science and Engineering, Vol.4, No.3, pp.165-172, 2001.

[14] A.G.Aissaoui, H.Abid and M.Abid : Robust Fuzzy sliding mode controller design for motor driver, Acta Electrotechnica et Informatica Vol. 9, No. 2, pp 64-71, 2009.

[15] Lon-Chen Hung and Hung-Yuan Chung : Hybrid Neural Sliding Mode Controller Design for a Robot Manipulator, Submitted to The Journal of Grey System Date: Sept 2004. 